\title{
Cisto dentígero em mandíbula: relato de caso
}

\section{Dentigerous cyst in mandible: case report}

Quiste dentígero em mandíbula: reporte de caso

Gustavo Paiva Custódio

ORCID: https://orcid.org/0000-0001-6893-369X Centro Universitário de Volta Redonda, Brasil E-mail: gustavopaivacustodio@gmail.com

Cesar Feitoza Bassi Costa

ORCID: https://orcid.org/0000-0002-5040-6602 Centro Universitário de Volta Redonda, Brasil

E-mail: cesar_bassi@hotmail.com

Carlos Henrique Silveira de Castro

ORCID: https://orcid.org/0000-0003-1583-0595

Universidade Federal da Bahia, Brasil

E-mail: carloshsilveirac@hotmail.com

Kaytlhen Kayllen Carvalho de Menezes

ORCID: https://orcid.org/0000-0002-6345-8874

Universidade Federal do Maranhão, Brasil

E-mail: menezes.kaytlhen@gmail.com

Isabela Pulino Padilha

ORCID: https://orcid.org/0000-0002-1699-4893

Universidade Nove de Julho, Brasil

E-mail: be.pulino@gmail.com

Thayná Cristina Ferreira Costa

ORCID: https://orcid.org/0000-0001-6752-7227

Universidade Federal de Juiz de Fora

E-mail: thayna.ferreira@estudante.ufjf.br

Isabela Gomes de Sena Ribeiro

ORCID: https://orcid.org/0000-0003-4657-1901

Universidade Católica de Brasília, Brasil

E-mail: isabelasenar@gmail.com

Dâmaris da Rocha Menezes

ORCID: https://orcid.org/0000-0002-1759-1084

Soberana Faculdade de Saúde de Petrolina e Vale do São João, Brasil

E-mail: damarisrmnz@gmail.com

Alef Vieira Galvão

ORCID: https://orcid.org/0000-0002-4066-224X

Centro Universitário Tocantinense Presidente Antônio Carlos, Brasil

E-mail: alefgalvao@gmail.com

Isabela Leandra Mendes Menezes

ORCID: https://orcid.org/0000-0002-6858-2292

Pitágoras de Belo Horizonte, Brasil

E-mail: isabelaleandramendes@gmail.com

Vittor Dorinato de Santana Sátiro

ORCID: https://orcid.org/0000-0002-7069-0807 Universidade Paulista, Brasi

E-mail: vittorodonto@gmail.com

Leonardo Gonzaga de Lima Vargas

ORCID: https://orcid.org/0000-0001-5745-8643

Universidade São Francisco, Brasil

E-mail: leoogonzaga1@ hotmail.com

Felipe Henrique Rech Payor

ORCID: https://orcid.org/0000-0002-3608-3771

Universidade São Francisco, Brasi

E-mail: felipepayor@hotmail.com

Lucas Ribeiro Modesto

ORCID: https://orcid.org/0000-0002-9950-7412

Universidade de Itaúna, Brasil

E-mail: 1ribeiro_07@outlook.com 


\author{
Matheus Almeida Barbosa \\ ORCID: https://orcid.org/0000-0002-3302-8056 \\ Universidade Católica de Brasília, Brasil \\ E-mail: matheusbarbosa13@gmail.com \\ José Igor da Silva \\ ORCID: https://orcid.org/0000-0002-1353-2642 \\ Faculdade Maurício de Nassau, Brasil \\ E-mail:sigor016@gmail.com
}

\begin{abstract}
Resumo
O cisto dentígero é identificado como uma cavidade revestida de epitélio odontogênico que circunda a coroa de um dente incluso, localizando-se principalmente na mandíbula e na região do terceiro molar inferior. Sua formação ocorre devido ao acúmulo de fluído, originado pela pressão exercida no folículo, por um dente que tenta erupcionar. É mais comum em indivíduos entre 10 e 30 anos de idade, havendo preferência por pacientes do sexo masculino. A lesão normalmente é assintomática, sendo descoberta em exames radiográficos de rotina. Radiograficamente observa-se uma área radiolúcida bem delimitada associada à coroa de um dente incluso. As opções de tratamento são enucleação, marsupialização e a descompressão seguida por enucleação. As hipóteses de diagnóstico inicial foram cisto dentigero, ceratocisto ou ameloblastoma unicistico. O objetivo deste trabalho é dissertar um caso clínico, de uma paciente, do sexo feminino, 32 anos de idade, melanoderma, que apresentava um cisto dentígero de grande extensão associado ao elemento dentário 38 incluso. A opção de tratamento abordada, foi de acordo com as características das lesões, foi instituída a enucleação com remoção do elemento envolvido e o acompanhamento clínico com exames radiográficos. Concluiu-se que diante da grande frequência, torna-se indispensável o conhecimento do cirurgião-dentista acerca das características clínico-radiográficas do cisto dentígero para que possam ser adequadamente diagnosticados e tratados. No acompanhamento radiográfico revela completa neoformação óssea local, sem sinal de recidiva da lesão.
\end{abstract}

Palavras-chave: Cisto dentígero; Enucleação; Saúde bucal.

\begin{abstract}
The dentigerous cyst is identified as a cavity lined with odontogenic epithelium that surrounds the crown of an impacted tooth, located mainly in the mandible and in the region of the lower third molar. Its formation occurs due to the accumulation of fluid, caused by the pressure exerted on the follicle, by a tooth that tries to erupt. It is more common in individuals between 10 and 30 years of age, with a preference for male patients. The lesion is usually asymptomatic, being discovered on routine radiographic examinations. Radiographically, a well-defined radiolucent area associated with the crown of an impacted tooth is observed. Treatment options are enucleation, marsupialization, and decompression followed by enucleation. The initial diagnosis hypotheses were dentigerous cyst, keratocyst or unicystic ameloblastoma. The objective of this work is to present a clinical case, of a female patient, 32 years old, black, who presented a dentigerous cyst of great extension associated to the tooth 38 included. The treatment option addressed was according to the characteristics of the lesions, enucleation was instituted with removal of the involved element and clinical follow-up with radiographic exams. It was concluded that, given the high frequency, it is essential for the dentist to know the clinical and radiographic characteristics of the dentigerous cyst so that they can be properly diagnosed and treated. Radiographic follow-up reveals complete local bone neoformation, with no sign of lesion recurrence.
\end{abstract}

Keywords: Dentigerous cyst; Enucleation; Oral health.

\title{
Resumen
}

El quiste dentígero se identifica como una cavidad revestida de epitelio odontogénico que rodea la corona de un diente impactado, ubicado principalmente en la mandíbula y en la región del tercer molar inferior. Su formación se produce por la acumulación de líquido, originado por la presión que ejerce sobre el folículo, un diente que intenta erupcionar. Es más común en individuos entre 10 y 30 años, con preferencia por los pacientes masculinos. La lesión suele ser asintomática y se descubre en los exámenes radiográficos de rutina. Radiográficamente, se observa un área radiolúcida bien definida asociada con la corona de un diente impactado. Las opciones de tratamiento son enucleación, marsupialización y descompresión seguidas de enucleación. Las hipótesis diagnósticas iniciales fueron quiste dentígero, queratoquiste o ameloblastoma uniquístico. El objetivo de este trabajo es presentar un caso clínico, de una paciente de sexo femenino, 32 años, de raza negra, que presentó un quiste dentígero de gran extensión asociado al diente 38 incluido. La opción de tratamiento abordada fue de acuerdo a las características de las lesiones, se instauró enucleación con remoción del elemento involucrado y seguimiento clínico con exámenes radiográficos. Se concluyó que, dada la alta frecuencia, es fundamental que el odontólogo conozca las características clínicas y radiográficas del quiste dentígero para que puedan ser debidamente diagnosticadas y tratadas. El seguimiento radiográfico revela una neoformación ósea local completa, sin signos de recidiva de la lesión.

Palabras clave: Quiste dentígero; Enucleación; Salud bucal. 


\section{Introdução}

O cisto dentígero (CD) é o tipo mais comum de cisto odontogênico de desenvolvimento e é comumente associado a coroa de um dente impactado ou não irrompido (Sindi, 2019). Observa-se que essa entidade patológica afeta mais pessoas do sexo masculino, leucodermas, entre a primeira e terceira década de vida (Souza et al., 2021; Önay et al., 2019). Além disso, é mais frequente em dentes mandibulares inclusos, geralmente terceiros molares (Cakarer et al., 2011; Contar et al., 2011; Chouchene et al., 2021; Santos et al., 2020).

Acredita-se que seu desenvolvimento é folicular, ocorrendo após o acúmulo de fluído entre o epitélio e o esmalte de um dente incluso. A coroa do dente em formação acaba por exercer uma pressão sobre o folículo dentário, com isso há obstrução venosa e transudação de líquido pelas paredes capilares, que irá desagregar a coroa e o folículo dentário e assim, formando o CD (Daneshvar \& Daneshvar, 2019; Souza et al., 2021; Önay et al., 2019).

Clinicamente, o cisto dentígero apresenta-se assintomático, sendo comumente descoberto durante um exame radiográfico de rotina ou devido à não erupção de um dente. A imagem radiográfica do espaço folicular maior que 5 mm de diâmetro é sugestiva desta lesão cística. A ausência de sintomatologia contribui para a sua evolução, gerando expansão indolor do osso afetado e assimetria facial, podendo comprometer as estruturas ósseas adjacentes (Benossi et al., 2019; Salama \& ElFetouh, 2020; Souza, 2020).

As características radiográficas apontam uma imagem radiolúcida unilocular, com margens definidas e frequentemente esclerótica, associada a coroa de um dente incluso. Quando associado a um processo infeccioso, as margens podem se apresentar indefinidas. O diagnóstico definitivo é obtido através do exame histopatológico, por meio de uma biopsia excisional ou incisional (Silva et al., 2021; Souza et al., 2021).

Histologicamente o CD apresentam-se com características semelhantes a outros cistos odontogênicos de desenvolvimento. O CD dispõe de uma cápsula de tecido conjuntivo frouxa e delgado, revestido por células epiteliais não ceratinizadas, composto por duas ou três camadas de células planas e cubóides. Quando há infecção, podem estar presentes células inflamatórias crônicas e o tecido conjuntivo é mais denso (Caliento et al., 2013; Carli et al., 2013; Souza et al., 2021).

Dentre as opções de diagnóstico diferencial encontra-se o ceratocisto odontogênico, fibro-odontoma ameloblástico, fibroma ameloblástico e ameloblastoma unicístico (Silva et al., 2020; Neville et. al., 2016; Önay et al., 2019). Embora seja raro, já foi relatado sobre a possibilidade de o revestimento de um cisto dentígero sofrer transformação neoplásica para um ameloblastoma. (Amorim et. al., 2021; Stringhini et al., 2018; Swantek et al., 2012).

O tratamento deve ser escolhido tendo como base alguns critérios importantes, como o tamanho do cisto, idade do paciente, proximidade de estruturas anatômicas, importância clínica com possibilidade de uso do dente envolvido e a manutenção de sua vitalidade pulpar (Carvalho \& Luna, 2016; Bhardwaj et al., 2016; Yu et al., 2020).

Com frequência, a principal abordagem para este cisto é a enucleação seguida pela remoção do dente incluso. Porém quando são cistos maiores pode-se fazer uma marsupialização após a enucleação, antes do tratamento definitivo. Pois assim evita maiores prejuízos as estruturas ósseas e adjacentes (Daneshvar \& Daneshvar, 2019; Sindi, 2019; Önay et al., 2019). Em geral o cisto dentígero apresenta prognóstico favorável, sendo raramente observado recidiva da lesão (Sindi, 2019; Souza et al., 2021; Silva et al., 2021).

O objetivo deste trabalho é relatar um caso clínico, de uma paciente, do sexo feminino, 32 anos, melanoderma, que apresentava um cisto dentígero de grande extensão associado ao elemento dentário 38 incluso, assim como evidenciar as características clínicas, radiográficas e histopatológicas da lesão. 


\section{Metodologia}

Refere-se a um relato de caso, caracterizado como um estudo exploratório, descritivo e qualitativo (Pereira et al., 2018).

Foram seguidos os princípios éticos de pesquisa envolvendo seres humanos, sendo preservada a identidade do voluntário e fornecido todos os esclarecimentos sobre o tratamento e após concordância do paciente a assinatura do Termo de Consentimento Livre Esclarecido (TCLE), consentindo o tratamento, a divulgação das imagens e dados coletados durante o atendimento clínico e os que constavam no prontuário, sendo que o mesmo poderia desistir da participação na pesquisa a qualquer instante.

\section{Relato de Caso}

Paciente gênero feminino, 32 anos de idade, melanoderma, compareceu ao ambulatório do Serviço de Cirurgia e Traumatologia Buco-maxilo-facial, com queixa de aumento de volume amolecido na região retromolar esquerdo. Durante a anamnese, relatou que havia um "inchaço dentro da boca há cerca de 7 meses", negava ser tabagista e etilista. Não foi observado qualquer alteração de ordem sistêmica ou local.

Ao exame clínico extra-oral, não foi observado nenhuma alteração. No exame intraoral, constataram-se na região de corpo e ramo mandibular esquerdo havia tumefação com amolecimento de tecido naquele local, sem sangramento e dor, e a mucosa apresentava coloração de aspecto escurecido (Figura 1). Logo, solicitou-se um exame radiográfico e tomográfico para planejamento do tratamento.

Figura 1: Região retromolar com tumefação e consistência amolecida.

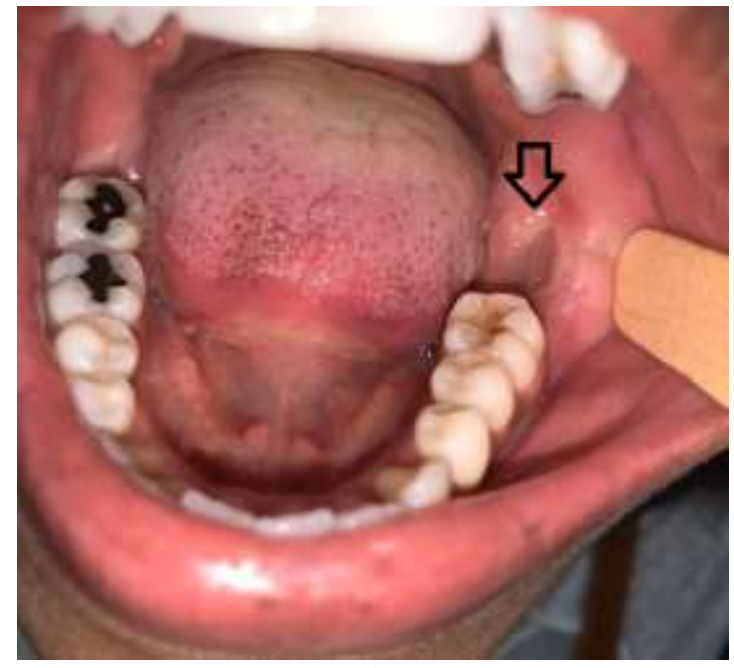

Fonte: Autores.

O exame imaginológico bidimensional, obtido através da radiografia panorâmica dos maxilares, evidenciava uma zona radiolúcida bem delimitada, unilocular, que envolvia o dente 38 que se encontrava totalmente incluso. Estendia-se do $1^{\circ}$ molar esquerdo (face distal) ao ramo mandibular do lado esquerdo (Figura 2). 
Figura 2: Extensão da lesão com envolvimento do elemento 38 incluso.

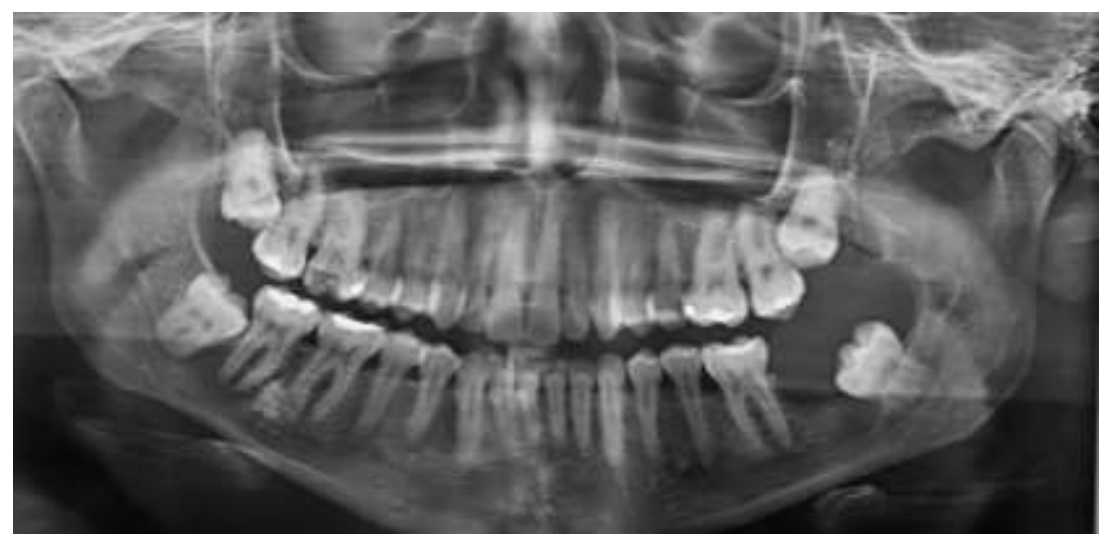

Fonte: Autores.

$\mathrm{Na}$ tomografia computadoriza, observou-se imagem hipodensa, de contornos bem definidos, associada ao elemento citado (Figura 3). Notava-se ainda que havia grande proximidade do nervo alveolar inferior esquerdo com a raiz do terceiro molar incluso, com possibilidade de parestesia (Figura 4). Com base nas características clínicas-radiográficas foi dada a hipótese diagnostica de cisto dentígero.

Figura 3: Lesão hipodensa associada a coroa do elemento 38.

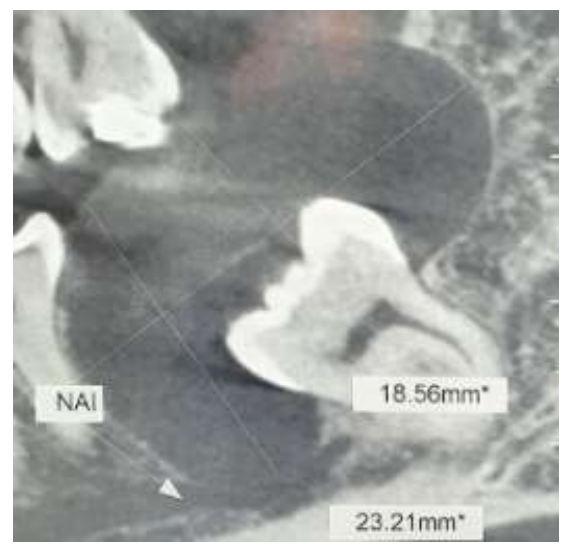

Fonte: Autores.

Figura 4: Proximidade da raiz com o nervo alveolar inferior esquerdo.

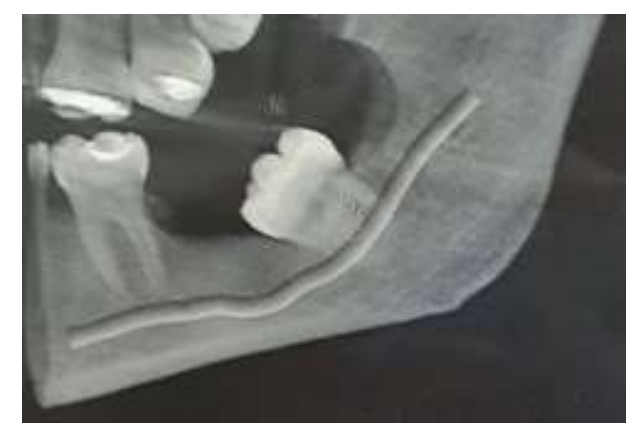

Fonte: Autores.

O tratamento cirúrgico realizado foi a enucleação do cisto por curetagem e extração do elemento 38 (terceiro molar inferior esquerdo), sob anestesia geral, após constatação da normalidade dos exames pré-operatórios solicitados (Figura 5 e 6 ). 
Research, Society and Development, v. 10, n. 12, e519101220782, 2021

(CC BY 4.0) | ISSN 2525-3409 | DOI: http://dx.doi.org/10.33448/rsd-v10i12.20782

Figura 5: Exposição e remoção da lesão cística e do elemento dentário envolvido.

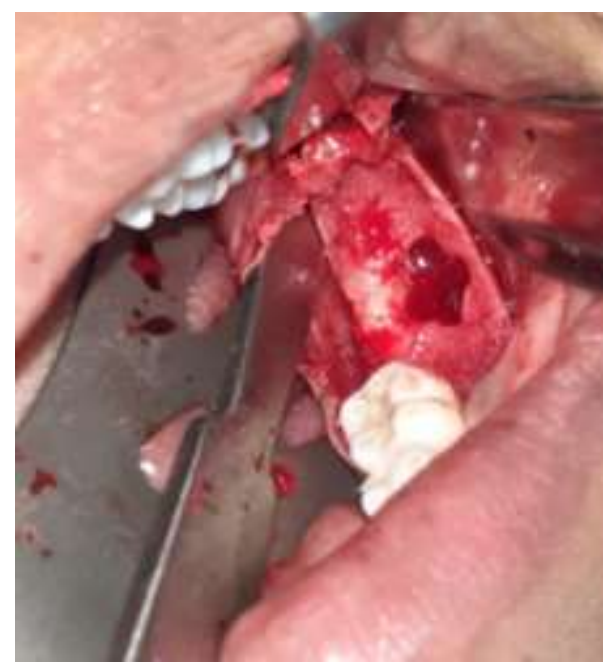

Fonte: Autores.

Figura 6: Elemento 38 removido com capsula cística.

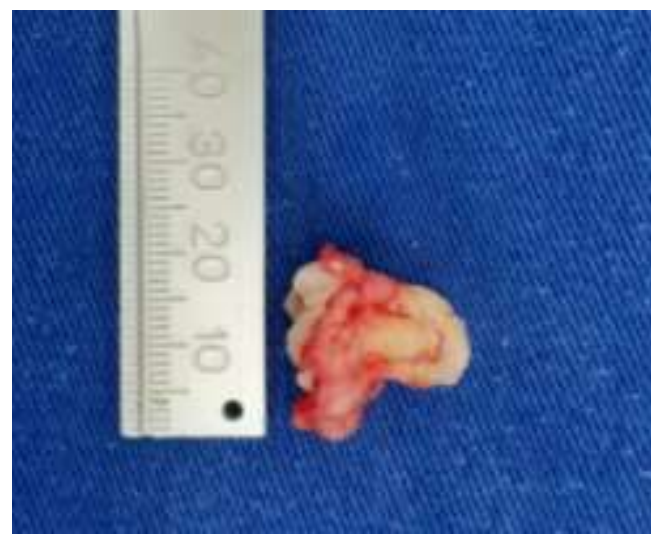

Fonte: Autores.

O cisto apresentava-se envolvido por uma cápsula espessa, o que tornou possível sua completa excisão (Figura 7). Apesar da proximidade, com o nervo alveolar inferior esquerdo conseguiu-se a preservação dos feixes vásculo-nervoso e de toda espessura da cortical óssea lingual. 
Figura 7: Cápsula cística.

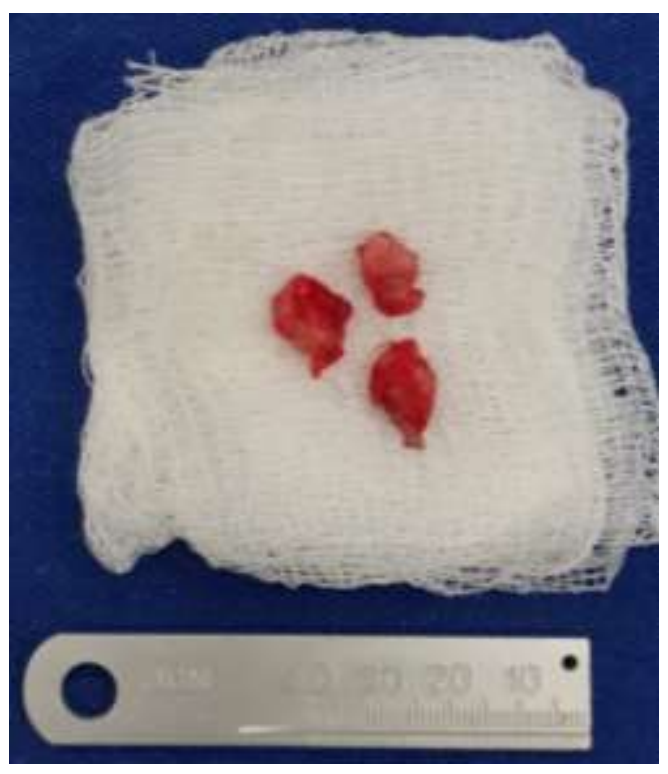

Fonte: Autores.

O dente e o fragmento após conservação em solução de Formol 10\% foram encaminhados para exame anatomopatológico. Após processamento histológico os cortes microscópicos revelaram fragmentos de mucosa sem alterações que se conecta com parede fibrosa espessa revista por epitélio escamoso não queratinizado ora atrófico ora com mais camadas celulares sem atipias, com discreta a moderada inflamação crônica. Contendo também remanescentes epiteliais odontogênicos e numerosos focos de calcificações distróficas, sendo compatível e confirmando a hipótese diagnóstica de cisto dentígero.

Foi realizada ainda, no mesmo tempo cirúrgico, a extração dos elementos 18, 28 e 48 (terceiros molares) que encontravam-se inclusos, por prevenção de problemas futuros como reabsorção radicular, doença periodontal, cistos e tumores odontogênicos (Figura 8).

Figura 8: Ausência dos terceiros molares.

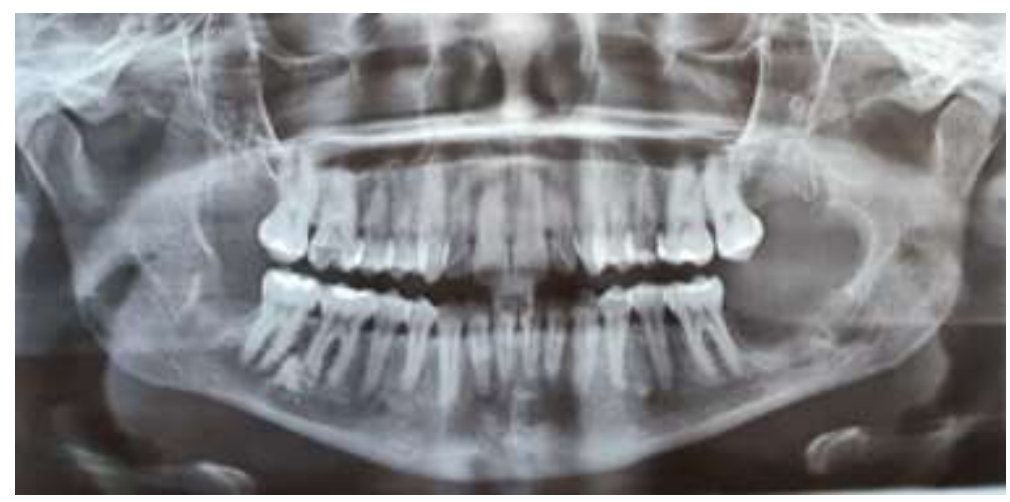

Fonte: Autores.

Foi realizada sutura e proservação da paciente. A cicatrização após alguns dias encontrava-se dentro dos padrões de normalidade, sem tumefação ou deiscência da sutura (Figura 9). 
Figura 9: Remoção de sutura e cicatrização adequada.

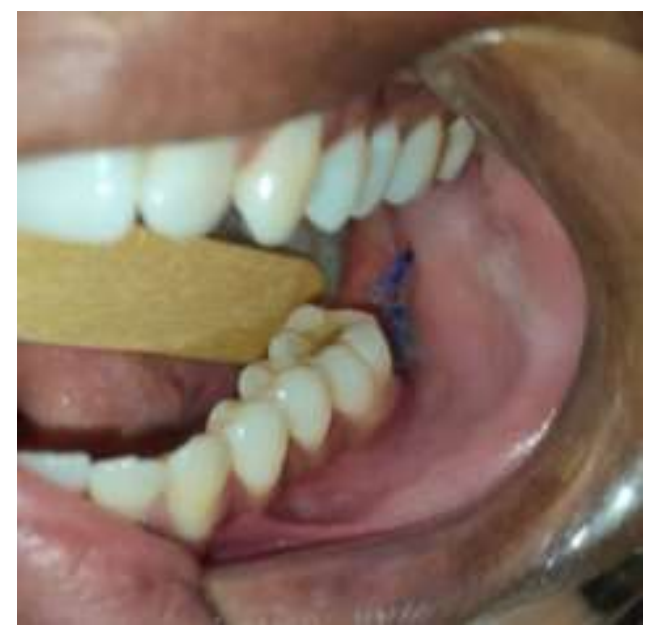

Fonte: Autores.

Durante o período de 7 meses, a paciente foi acompanhada e não houve qualquer intercorrência no pós-operatório, nem parestesia ou outro sinal de recidiva da lesão. O dente próximo à lesão apresentou teste de vitalidade positivo.

\section{Discussão}

Apesar de ser mais frequente em dentes mandibulares inclusos, geralmente terceiros molares, pode acometer dentes maxilares, como os molares, caninos, pré-molares não irrompidos e dentes supranumerários (Cakarer et al., 2011; Contar et al., 2011; Önay et al., 2019). Além disso, este cisto pode ser associado com a displasia cleidocraniana e a síndrome de MaroteauxLamy (Chouchene et al., 2021; Peruchi et al., 2001; Sindi, 2019;). No presente caso, foi observado em um terceiro molar inferior incluso, corroborando com a grande parte da literatura fundada.

Quanto à associação entre o sexo, há leve prevalência para o gênero masculino, leucodermas, entre a primeira e terceira década de vida (Silva et. al., 2021; Neumann et al., 2021; da Silva et al., 2020; Neville et. al., 2021). Neste estudo, foi abordado uma paciente do sexo feminino e melanoderma, contrariando-se da literatura pesquisada. A faixa etária soma-se a literatura, visto que a mesma possui 32 anos.

A etiologia dos cistos de desenvolvimento é desconhecida, porém essas lesões não são resultantes de uma reação inflamatória, diferindo assim dos cistos de inflamação (Neumann et al., 2021; Neville et. al., 2016). Na maioria dos casos o cisto dentígero é assintomático e não apresenta repercussão clínica (Contar et al., 2011). No relato de caso abordado, a paciente também não apresentava dor. Entretanto, lesões de grandes dimensões podem causar expansão do osso alveolar e sensibilidade local. Tem como característica o crescimento lento e expansivo, podendo causar o deslocamento do dente acometido e dentes vizinhos, reabsorção de radicular e deslocamento do canal mandibular (Daneshvar \& Daneshvar, 2019; Neumann et al., 2021; 2012; Silva et al., 2020; Swantek et al., 2012).

O diagnóstico diferencial de todas as lesões é feito por meio da punção aspirativa, tendo em vista que grandes lesões podem ser tumores odontogênicos e não cistos como se espera, sendo a aspiração de líquido no interior da lesão um grande indicativo de cisto. A biópsia incisional antes do tratamento definitivo também deve ser feita a fim de excluir outras hipóteses diagnósticas como ceratocisto odontogênico e o ameloblastoma unicístico (Peruchi et al., 2001; Safira et al., 2009; Vaz et al., 2010).

Para que ocorra um prognóstico favorável e rara recidiva, a escolha do tratamento deve ser realizada com cautela e priorizando os fatores do paciente. O tratamento mais comum para o cisto dentígero é a enucleação cuidadosa do cisto junto 
com a remoção do dente não erupcionado, porém, existem outras opções como a marsupialização ou descompressão seguida por enucleação (Neville et. al., 2016; Neumann et al., 2021; Silva et al., 2020).

A avaliação de alguns aspectos do paciente, como idade, estado de saúde geral, colaboração, extensão e localização da lesão, deve ser levada em conta para a escolha do melhor tratamento (Cakarer et al., 2011; Chouchene et al., 2021). As opções de tratamento mais utilizadas são enucleação, marsupialização, ou descompressão seguida por enucleação (Contar et al., 2011; Salama \& ElFetouh, 2020). A técnica de eleição para esse caso foi a enucleação com remoção do elemento envolvido.

\section{Considerações Finais}

Por fim, é válido ressaltar a importância do conhecimento quanto as alterações patológicas que acometem a cavidade oral, para que seja realizado o diagnóstico correto e consequentemente o tratamento adequado. O sinal clínico bastante evidente do cisto dentígero é a imagem radiolúcida descoberta em exame de rotina envolvendo um dente incluso, logo, evidencia-se a importância de consultas periódicas ao dentista. O diagnóstico é estabelecido principalmente através do exame clínico, radiográfico e histopatológico.

A técnica cirúrgica empregada é segura tomando os devidos cuidados. É necessário planejamento cirúrgico cauteloso para remoção da lesão, levando em consideração a extensão da lesão, relação do cisto com estruturas anatômicas importantes. Sendo essencial para o sucesso do tratamento do cisto.

Ademais, sugere-se a realização de mais pesquisas para comprovar a efetividade de outros meios de tratamentos não cirúrgicos para o cisto dentígero e meios cirúrgicos associados a outras técnicas pouco relatadas em literatura.

\section{Referências}

Amorim, K. S., Dantas, A. C. G. C., Nascimento, A. M. da S., Rios, M. L. S., Júnior, R. L. de A., \& Souza, L. M. de A. (2021). Cisto dentígero com transformação ameloblástica. Rev Cubana Estomatol, 58(1), e3028.

Bang, K. O., Yadav, S. R., Shenoi, R., Budhraja, N., \& Ingole, P. (2021). Bilateral Non-Syndromic Dentigerous Cyst - Rare Case Report and Literature Review. Journal Pre-proof, 2667-1476.

Bhardwaj, B., Sharma, S., Chitlangia, P., Agarwai, P., Bhamboo, A. \& Rastogi., K. (2016). Mandibular Dentigerous Cyst In A 10-year-old Child. International Journal of Clinical Pediatric Dentistry, 9(3), 281-284.

Cakarer, S., Selvi F., Isler S. C. \& Keskin C. (2011). Decompression, enucleation, and implant placement in the management of large dentogerous cyst. $J$ Craniofac Surg. 922-924.

Caliento, R., Mannarino, F. S. \& Hochuli-Vieira, E. (2013). Cisto dentígero: modalidades de tratamento. Rev Odontol. Unesp, 6(42), 458-462.

Chouchene, F., Ameur, W. B., Hamdi, H., Bouenba, M., Masmoudi, F., Baaziz, A., Maatouk, F., \& Ghedira, H. (2021). Conservative Approach of a Dentigerous Cyst. Case reports in dentistry, 2(45), 22-8.

Carli, M. L., Pedreira, F. R. O., Guimarães, E. P., Dias, N. N. O., Pereira, A. A. C., \& Hanemann, J. A. C. (2013). Conservative surgical-orthodontic treatment of a young patient with a dentigerous cyst. Rev. Odontol. Unesp, 5(42), 217-222.

Carvalho, I. K. F. \& Luna, A. H. B. (2016). Spontaneous Eruption of Premolar Associated with a Dentigerous Cyst. Case Reports In Dentistry, 5(45), 1-5.

Contar C. M. M., Thomé, C. A., Pompermayer, A., Sarot J. R., Vinagre, R. O., Machado, M. A. N. (2011). Marsupialization of dentigerous cyst: Report of a case. J Maxillofac Oral Surg, 26(3), 1-3

Daneshvar, S. H., \& Daneshvar, M. M. (2019). Mandibular dentigerous cyst in a 10-year-old patient. Pediatric Dental Journal, 29(3), 157-160.

Jung, E., Baek, J. \& Leem, D. (2014). Decompression Device Using a Stainless Steel Tube and Wire for Treatment of Odontogenic Cystic Lesions: A Technical Report. Maxillofacial Plastic And Reconstructive Surgery, 6(36), 308-310.

Neville, B.W., Damm, D.D., Allen, C.M., Chi, A.C. (2016). Patologia Oral \& Maxilofacial, $4^{o}$ edição. Grupo Editorial Nacional.

Neumann, B. L., Perotto, S. T., Gabriel, A. D. F., Zanella, V. G., Silveira, F. M., Kroef, R. G., \& Martins, M. D. (2021). Cistos e tumores odontogênicos: estudo retrospectivo dos casos diagnosticados no período de 10 anos em um hospital no sul do Brasil. Revista da Faculdade de Odontologia de Porto Alegre. Porto Alegre. Vol. 62, n. 1 (2021), p. 56-62.

Önay, Ö., Süslü, A. E., \& Yılmaz T. (2019). Huge dentigerous cyst in the maxillary sinus: a rare case in childhood. Turk Arch Otorhinolaryngol, 57(1), 54-56. 
Research, Society and Development, v. 10, n. 12, e519101220782, 2021 (CC BY 4.0) | ISSN 2525-3409 | DOI: http://dx.doi.org/10.33448/rsd-v10i12.20782

Pereira, A. S., Shitsuka, D. M., Parreira, F. J. \& Shitsuka, R. (2018). Metodologia da pesquisa científica.[ebook].SantaMaria.Ed.UAB/NTE/UFSM.https://repositorio.ufsm.br/bitstream/handle/1/15824/LicComputacao_Metodologia PesquisaCientifica.pdf?sequence $=1$.

Peruchi, C. M. S., Silva, E. B., Borsatto, M. C., Pardini, L. C., \& Gosuen, L. C. (2001). Tratamento alternativo não cirúrgico de cisto dentígero em criança: relato de caso. Robrac, 10(29), 9-13.

Safira, L. C., Paim, J. M., Jr, B. C., Queiroz, C. S., Oliveira, T. B., Ramalho, L. P., \& Sarmento, V. A. (2009). Cisto dentígero em mandíbula: relato de caso clínico. $R$. Ci. méd. biol., 8(2), 225-229.

Santos, A. M. S., Mendes, B. C, Statkievicz, C., Lima, T. J., Tormes, A. K., \& Hochuli-Vieira, E. (2020). Cisto dentígero e Osteomielite com periostite proliferativa: uma atualização diagnóstica. Pesquisa, Sociedade e Desenvolvimento, 9 (7), e866975020.

Salama, A. A., \& Abou-ElFetouh, A. (2020). Marsupialization and functional obturator placement for treatment of dentigerous cyst in child: A successful blend. Oral and Maxillofacial Surgery Cases, 2(34) 19-23.

Silva, M. P., Zenatti, R., Conci, R., Junior, E. Á. G., Magro, N. E., \& Griza, G. L. (2021). Enucleação de extenso cisto dentígero em ambiente ambulatorial: relato de caso. Brazilian Journal of Health Review, 4(3), 10606-10619.

Silva, I. D., Lobo, F., Silva, B. G., Souza Tolentino, E., Iwaki, L. C. V., \& da Silva, M. C. (2020). Cistos odontogênicos inflamatórios e de desenvolvimento: estudo observacional e retrospectivo de 23 anos. Revista da Faculdade de Odontologia de Porto Alegre, 61(1), 4-10.

Sindi, A. M. (2019). Bilateral mandibular dentigerous cysts presenting as an incidental finding: a case report. Am J Case Rep, $20,1148-1151$.

Souza, B. C. (2020). Cisto dentígero de grande dimensão em maxila: relato de caso. Revista Journal of Health-ISSN 2178-3594, 1(1).

Stringhini J. E., Galvani E. C., Zenatti R., Stang B. \& Becker G. A. (2018). Opções cirúrgicas para tratamento de cisto dentígero: série de casos. Rev Assoc Paul Cir Dent, 72(4), 624-30.

Swantek J. J., Reyes M. I., Grannum R. I. \& Ogle O. E. (2012). A technique for long term decompression of large mandibular cysts. J Oral Maxillofac Surg, 70, 856-859.

Vaz, L. G. M., Rodrigues, M. T. V., \& Júnior, O. F. (2010). Cisto dentígero: características clínicas,radiográficas e critérios para o plano de tratamento. Rgo, Porto Alegre, 1(58), 127-130.

Yu, Y., Li, m., Zhou, Y., Shi, Y., Zhang, W., Son, G., Ge, J., Zhao, J., Zhang, Z., Ye, D., Yang, c., \& Wang, S. (2020). A ativação de células-tronco mesenquimais promove a formação de novo osso dentro do cisto dentígero. Stem Cell Research \& Therapy, 11-476. 